\title{
Extrathyroidal Extension into Strap Muscles Does Not Lower Overall Survival or Independently Predict Recurrence in Papillary Thyroid Cancer
}

\section{Benjamin J. Gigliotti}

Division of Endocrinology, Diabetes and Metabolism; Department of Medicine; University of Rochester School of Medicine \& Dentistry; Rochester, New York, U.S.A.

Review of: Li G, Li R, Song L, Chen W, Jiang K, Tang H, Wei T, Gong R, Li Z, Lei J, Zhu J 2019 Implications of extrathyroidal extension invading only the strap muscles in papillary thyroid carcinomas. Thyroid. Epub 2019 Dec 13. PMID: 31830859.

\section{SUMMARY}

\section{Background}

The 8th edition of the American Joint Committee on Cancer (AJCC) staging system for differentiated thyroid cancers, considers tumors $>4$ centimeters to be stage "T3a," and tumors that extend outside the thyroid and grossly invade strap muscles are designated "T3b" (1). This is a departure from prior editions, which did not subdivide the stage T3 category and which included microscopic extrathyroidal extension (ETE) in the definition of T3 disease (2). While gross invasion of major neck structures carries significant morbidity, microscopic ETE does not appear to be an independent predictor of recurrence or mortality. However, there is a paucity of data on the prognostic significance of isolated gross invasion into local neck strap muscles. This study (3) sought to assess the clinical significance of extrathyroidal extension in papillary thyroid carcinoma (PTC), with a focus on isolated strap muscle invasion in stage T3b thyroid cancer tumors.

\section{Methods}

This retrospective cohort study included 4045 patients with papillary thyroid carcinoma (PTC) who underwent surgery at the Thyroid and Parathyroid Surgery Center in West China Hospital, Chengdu, China between 2011 and 2016.
Exclusion criteria included age $<18$ or $>80$ years, multiple pathologic diagnoses, positive tumor margins, and incomplete records. Patients were divided into four groups depending on the extent of ETE: group 1, no ETE; group 2),ETE into perithyroidal tissue, group 3, ETE into the sternohyoid, sternothyroid, thyrohyoid, or omohyoid "strap" muscles ("T3b" group), and group 4,ETE beyond the strap muscles into soft tissue or other local structures. The median follow up was 32 months (range, 12-63). The clinicopathologic criteria of each group, overall survival, and recurrence-free survival were assessed. Recurrent/persistent disease was defined as any tumor or distant metastases detected by imaging, abnormal thyroglobulin levels, or rising antithyroglobulin titers.

\section{Results}

Of the 4045 patients studied, 373 (9.2\%) had ETE into strap muscles. As compared with tumors without ETE, invasion into the strap muscles was associated with larger size $(18.4 \pm 10.6 \mathrm{~cm}$ vs. $11.2 \pm 8.1 \mathrm{~cm}, \mathrm{P}=0.009)$, multifocality $(33.4 \%$ vs. $27.1 \%, P=0.03)$, lymph node involvement $(65.2 \%$ vs. $45.2 \%, P=0.011$ ), and distant metastases (HR, 9.47; $\mathrm{P}<0.001$ ). Recurrence occurred in $22.5 \%$ of patients. Recurrence-free survival was lower for 

Does Not Lower Overall Survival or Independently Predict Recurrence in Papillary Thyroid Cancer

T3b tumors than for no ETE (HR, 1.89; $P=0.017)$, but there was no difference in cancer-specific death $(0.27 \%$ vs. $0.22 \%, P=0.433)$ or overall survival. However, on univariate analysis, T3b status was not independently associated with increased recurrence $(P=0.107)$. For comparison, tumors with gross ETE beyond the strap muscles had significantly poorer outcomes in all of the aforementioned measures, whereas tumors with minimal ETE into perithyroidal tissue showed no significant difference as compared with tumors with no ETE.

\section{Conclusions}

In adults ages 18 to 80 years with PTC, isolated extrathyroidal extension into neck strap muscles is associated with increased tumor size, multifocality, lymph node involvement, distant metastases, and a decrease in recurrence-free survival, but not a decrease in overall survival. However, the negative effect on recurrence-free survival was not seen after adjusting for associated clinicopathologic factors (especially age, male sex, tumors $>2 \mathrm{~cm}$, and lymph node metastases).

\section{COMMENTARY}

The 8th edition of the AJCC system has led to significant downstaging for many patients with differentiated thyroid cancer, largely through eliminating upstaging due to N1 disease and microscopic/ minimal perithyroidal ETE (4). The updated staging system also entails an increased age cutoff defining "older" patients (i.e., from 45 to 55 years) and a poorer prognosis with more advanced disease (4). A more parsimonious definition of clinically significant ETE is impactful. For example, a 58-year-old woman with isolated invasion of strap muscles was considered stage IV on December 31, 2017, but stage II on January 1, 2018, when the 8th edition went into effect. It is worth noting that the new T3a (tumors > $4 \mathrm{~cm}$, no ETE) and T3b (isolated gross strap muscle invasion) partitions confer the same effect on overall stage.

It is widely accepted that gross invasion of major neck structures negatively impacts risk of recurrence or mortality in PTC (5). Conversely, there is convincing evidence that microscopic extrathyroidal extension is not a predictor for overall survival for most patients, although it is arguable whether there is a small impact on recurrence risk (6). Debate often arises from the considerable difficulty in standardizing the definition of minimal ETE, since the thyroid does not have a complete capsule, and high interobserver variability exists in distinguishing "bulging into" versus "invasion of" soft tissues (7). However, the prognostic significance of gross ETE into skeletal muscle alone (without involving the larynx, trachea, esophagus, recurrent laryngeal nerve, subcutaneous tissue, etc.) has only recently been investigated. The aforementioned results are largely in agreement with three other recent studies from the United States and Korea that showed no independent clinical impact of isolated strap muscle invasion (8-10); it is, however, not surprising that the studies differ in the impact of minimal ETE for the reasons mentioned above.

The strengths of this study include the large number of patients, thoughtful clinical and histologic variables, and a low frequencies of incomplete records and follow-up. Limitations include the usual criticisms about single-center retrospective studies, the loss of significance for some variables when using multiple-comparison correction, and most importantly, a relatively short follow-up period 

Does Not Lower Overall Survival or Independently Predict Recurrence in Papillary Thyroid Cancer

(median, 32 months), which particularly limits assessment of overall survival, since the vast majority of PTCs are indolent. There was a somewhat high prevalence of $\mathrm{T} 3 \mathrm{~b}$ disease, and the rate of recurrence was higher than average (22.3\%) during the short period of study follow-up. However, this study clearly bolsters growing evidence that isolated gross strap muscle invasion does not predict recurrence or mortality, independent of traditional clinicopatho- logic risk factors. This evidence supports the AJCC 8th edition's co-categorization of large tumors and isolated strap muscle invasion as T3, along with the downgrade to prognostic stage II for T3 disease. If tumors with isolated strap muscle invasion behave similarly to large intrathyroidal tumors, it is perhaps reasonable to consider a more conservative management approach, which is growing increasingly standard in contemporary thyroid cancer care.

\section{References}

1. American Joint Committee on Cancer 2018 AJCC cancer staging manual, 8th ed. New York: Springer.

2. American Joint Committee on Cancer 2011 AJCC cancer staging manual, 7th ed. New York: Springer.

3. Li G, Li R, Song L, Chen W, Jiang K, Tang H, Wei T, Gong R, Li Z, Lei J, Zhu J 2019 Implications of extrathyroidal extension invading only the strap muscles in papillary thyroid carcinomas. Thyroid. Epub 2019 Dec 13.

4. Tuttle RM, Haugen B, Perrier ND 2017 Updated American Joint Committee on Cancer/Tumor-NodeMetastasis Staging System for Differentiated and Anaplastic Thyroid Cancer (eighth edition): what changed and why? Thyroid 27:751-756.

5. Ito Y, Tomoda C, Uruno T, Takamura Y, Miya A, Kobayashi K, Matsuzuka F, Kuma K, Miyauchi A 2006 Prognostic significance of extrathyroid extension of papillary thyroid carcinoma: Massive but not minimal extension affects the relapse-free survival. World J Surg 30:780-786.

6. Nixon IJ, Ganly I, Patel S, Palmer FL, Whitcher MM, Tuttle RM, Shaha AR, Shah JP 2011 The impact of microscopic extrathyroid extension on outcome in patients with clinical T1 and T2 well-differentiated thyroid cancer. Surgery 150:1242-1249.
7. Su HK, Wenig BM, Haser GC, Rowe ME, Asa SL, Baloch Z, Du E, Faquin WC, Fellegara G, Giordano T, et al. 2016 Inter-observer variation in the pathologic identification of minimal extrathyroidal extension in papillary thyroid carcinoma. Thyroid 26:512-517.

8. Park SY, Kim HI, Kim JH, Kim JS, Oh YL, Kim SW, Chung JH, Jang HW, Kim TH 2018 Prognostic significance of gross extrathyroidal extension invading only strap muscles in differentiated thyroid carcinoma. Br J Surg 105:1155-1162.

9. Amit M, Boonsripitayanon M, Goepfert RP, Tam S, Busaidy NL, Cabanillas ME, Dadu R, Varghese J, Waguespack SG, Gross ND, et al. 2018

Extrathyroidal extension: Does strap muscle invasion alone influence recurrence and survival in patients with differentiated thyroid cancer? Ann Surg Oncol 25:3380-3388.

10. Song E, Kim WW, Jeon MJ, Sung TY, Song DE, Kim TY, Chung KW, Kim WB, Shong YK, Hong SJ, et al. 2019 Clinical significance of gross invasion of strap muscles in patients with 1- to 4-cm-sized papillary thyroid carcinoma undergoing lobectomy. Ann Surg Oncol 26:4466-4471. 